\title{
Drug-Induced Liver Injury in a Patient with Nonsmall Cell Lung Cancer after the Self-Administration of Fenbendazole Based on Social Media Information
}

\author{
Teppei Yamaguchi Junichi Shimizu Yuko Oya Yoshitsugu Horio \\ Toyoaki Hida \\ Department of Thoracic Oncology, Aichi Cancer Center Hospital, Nagoya, Japan
}

Keywords

Fenbendazole $\cdot$ Nonsmall cell lung cancer $\cdot$ Liver injury $\cdot$ Social media $\cdot$ Pembrolizumab

\begin{abstract}
Fenbendazole is a benzimidazole anthelmintic agent, with a broad antiparasitic range in animals such as dogs and pigs. The agent is also reported to exert antitumor effects and inhibit microtubule-associated tubulin polymerization, but its safety and tolerability profile in humans remains unclear. An 80-year-old female patient with advanced nonsmall cell lung cancer (NSCLC) was started on pembrolizumab monotherapy. The patient experienced severe liver injury 9 months later. An interview with her and her family revealed that she had been taking fenbendazole for a month, solely based on social media reports suggesting its effectiveness against cancer. After discontinuation of the self-administration of fenbendazole, the patient's liver dysfunction spontaneously resolved. The antitumor inhibitory effects of fenbendazole have been reported; however, she did not experience tumor shrinkage. This is the first case report of a patient with advanced NSCLC who self-administered the anthelmintic, fenbendazole. Twitter and Facebook are online social media platforms which have been constructively used to exchange information among cancer patients. However, sources of medical information on these platforms are often unproven, and it is difficult for nonmedical professionals to accurately select and filter complex medical information. Physicians should enquire patients about self-administration of orally ingested products, including dietary supplements, herbs, or bioactive compounds, in cases of unexpected adverse reactions.
\end{abstract}

\section{Karger $\stackrel{2}{\circ}$}




\section{Introduction}

Fenbendazole (methyl $N$-(6-phenylsulfanyl- $1 H$-benzimidazol-2-yl) carbamate) is a benzimidazole compound with broad antiparasitic spectrum use in various animals [1]. The agent exerts anthelmintic activities by binding to $\beta$-tubulin microtubule subunits and disrupting polymerization [2]. Recently, benzimidazole compounds, including fenbendazole, have been reported to have antitumor activities, via tubulin binding [3, 4]. Fenbendazole is considered a promising therapeutic agent for several cancers; however, its safety and tolerability in humans has not yet been clarified, although safety data exist for various animals [1].

Twitter and Facebook are online social media platforms which facilitate social networks between individuals. They are popular and have now spread to every corner of the society. It has been observed that the information diffusion power of social media is much greater than conventional media such as newspapers, magazines, and TV. Social networking sites have made it easy for nonmedical individuals to disseminate complex medical information; however, this involves the risk of spreading unproven medical facts. Here, we report the case of a patient with advanced nonsmall cell lung cancer (NSCLC) who obtained information on the antitumor activity of fenbendazole via social media. The patient self-administered fenbendazole orally and subsequently experienced severe liver injury.

\section{Case Presentation}

In October 2018, an 80-year-old woman was admitted to Aichi Cancer Center Hospital with a tumor in the upper lobe of her right lung. She underwent a transbronchial lung biopsy and was diagnosed with lung adenocarcinoma. She had stage IVB (cT4N2M1c) with brain metastases. Sequent analyses and the programmed death-ligand 1 (PD-L1) IHC 22C3 pharmDx assay (Dako North America) revealed the tumor had a KRAS G12A mutation, and PD-L1 expression as evaluated by the tumor proportion score was $80 \%$. In November 2018, we initiated PD-1 inhibitor treatment, that is, pembrolizumab monotherapy. Three months later, a CT scan showed a partial tumor response and decreased carcinoembryonic antigen (CEA) levels. In February 2019, CEA levels began to increase; however, a CT scan showed stable disease, and treatment with pembrolizumab monotherapy was continued. When she was next seen on August 13, 2019, blood tests showed elevated aspartate transaminase (AST) levels at $386 \mathrm{U} / \mathrm{L}$, alanine aminotransferase (ALT) at $487 \mathrm{U} / \mathrm{L}$, and total bilirubin at $1.3 \mathrm{mg} /$ $\mathrm{dL}$, although her liver function 3 weeks ago was at the upper limit of normal (ULN), with an AST level of $24 \mathrm{U} / \mathrm{L}$ (ULN $\leq 33 \mathrm{U} / \mathrm{L}$ ), ALT of $16 \mathrm{U} / \mathrm{L}$ (ULN $\leq 27 \mathrm{U} / \mathrm{L}$ ), and total bilirubin of 0.8 $\mathrm{mg} / \mathrm{dL}$ (ULN $\leq 1.2 \mathrm{mg} / \mathrm{dL}$ ). Therefore, the patient was admitted to our hospital on an emergency basis (Fig. 1). Her physical findings and vital signs were unremarkable. She had no known history of drug or food allergies. Initially, we suspected liver injury as an immunerelated adverse event. For this reason, pembrolizumab monotherapy was temporarily discontinued, but after interviews with her family, it was discovered she had been taking oral fenbendazole since early July 2019. She and her family had become afraid of cancer progression since her CEA levels had increased. She had purchased and started taking oral fenbendazole, which was marketed as an anthelmintic for dogs, based on information from social media sites stating it was effective against cancer. She stated her fenbendazole schedule consisted of $1 \mathrm{~g} /$ day PO for 3 days, followed by 4 days off, and this schedule had been maintained for approximately 1 month from early July until her August visit. We stopped fenbendazole administration immediately, and her liver function parameters gradually improved. Subsequently, pembrolizumab was recommenced, and there was no relapse in liver dysfunction. Her CEA levels rose slightly after starting fenbendazole (i.e., $52.7-76.7 \mathrm{ng} / \mathrm{mL}$ ), and a CT scan

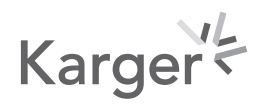




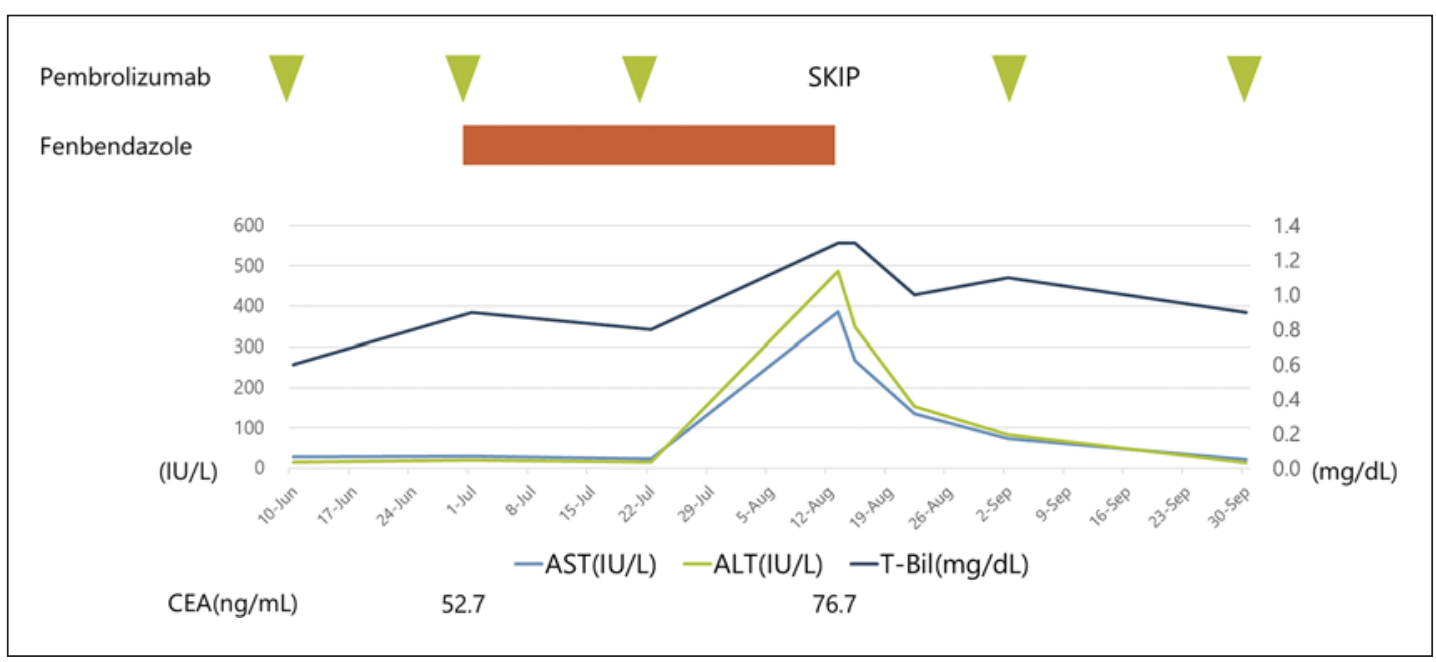

Fig. 1. Liver enzyme and CEA levels. Fenbendazole at $1 \mathrm{~g}$ per day PO for 3 days, followed by 4 days off, was repeated by the patient for approximately 1 month from early July until August 9, 2019. On August 13, blood tests showed elevated AST at $386 \mathrm{U} / \mathrm{L}$, ALT at $487 \mathrm{U} / \mathrm{L}$, and total bilirubin at $1.3 \mathrm{mg} / \mathrm{dL}$. After fenbendazole discontinuation, the patient's liver dysfunction gradually improved. AST, aspartate transaminase; ALT, alanine aminotransferase; CEA, carcinoembryonic antigen.
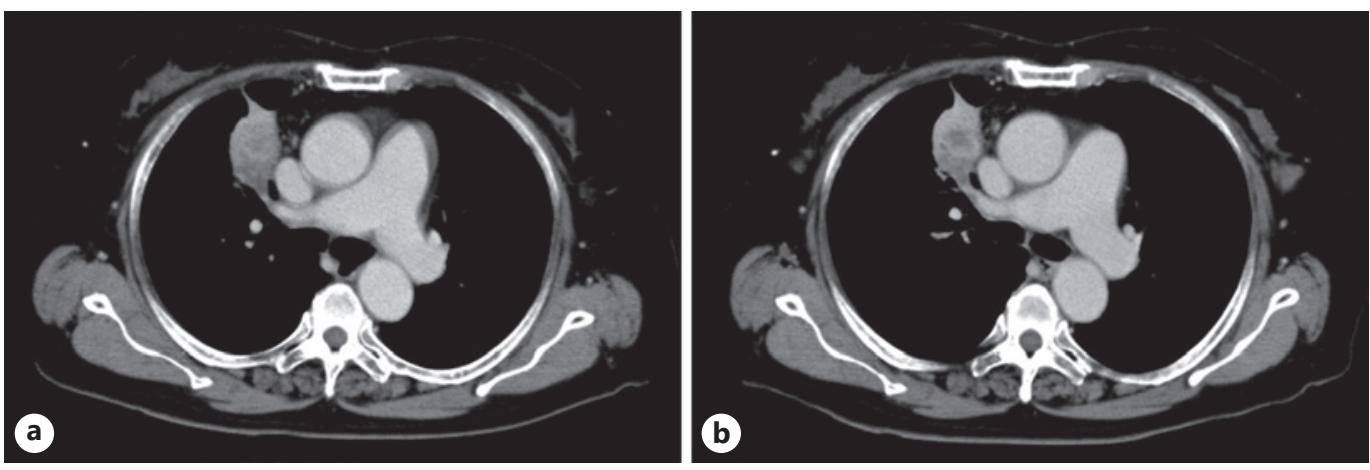

Fig. 2. Chest CT images during fenbendazole administration. a CT findings just before the oral initiation of fenbendazole on July 1, 2019. b CT findings on August 15, 2019, after taking fenbendazole for approximately 1 month.

on August 15 showed no change in tumor size (Fig. 2). Her AST, ALT, and total bilirubin levels had normalized on September 30, and on November 25, 2019, pembrolizumab monotherapy was terminated due to increased tumor size in the right upper lobe of her lung. The causality assessment evaluation was performed by using the Nranjo Adverse Drug Reaction Probability Scale [5]. The result was 6 points, indicating a "probable" adverse reaction to fenbendazole (see online suppl. Table 1 at www.karger.com/doi/10.1159/000516276).

\section{Discussion}

Fenbendazole is widely used as an antiparasitic agent against various gastrointestinal parasites, such as pinworms, giardia, roundworms, hookworms, Taenia solium, and pulmonary paragonimiasis [6-13]. The agent is used in several animal species, that is, dogs, cats, rabbits, 
rats, horses, sheep, and cattle [6-15]. Fenbendazole exerts polymerization inhibitory effects toward tubulin, which are polymers that make up microtubules; these form part of the cytoskeleton and provide structure and shape to cells [3]. Cytotoxic anticancer agents which act on microtubules include vinca alkaloids (vinblastine, vincristine, vinorelbine, and vindesine) and taxanes (paclitaxel and docetaxel). Fenbendazole has been reported to have antitumor effects similar to cytotoxic anticancer agents [3]. In recent years, several studies have reported the in vitro and in vivo (animal models) antitumor effects of fenbendazole. Dogra et al. [4] reported that upon transplantation of human lung adenocarcinoma (A549) cells into female athymic nu/nu mice, $1 \mathrm{mg} /$ mouse fenbendazole was orally administered every 2 days for 12 days, resulting in a marked shrinkage of tumor size and weight. Another study reported that methiazole and fenbendazole suppressed RAS-related signaling pathway expression in lung cancer cells with a KRAS mutation [16]. These data indicated that fenbendazole was a new microtubule inhibitor with antitumor activity, and it exerted inhibitory activities toward the RAS-related signaling pathway. Thus, it was potentially a promising therapeutic agent acting against various cell pathways to effectively eliminate tumors; however, the agent had not been clinically proven for the treatment of human tumors.

In many species, fenbendazole is only partly absorbed from the intestine after oral administration and is converted to the active compounds, oxfendazole and fenbendazole sulfone $[17,18]$. Since the 1970s, fenbendazole has been used as an anthelmintic drug for animals, but its role in humans has not been clarified due to the lack of phase 1 clinical trials investigating safety, tolerability, and optimal doses for humans. With regard to oxifendazole, which is an activated fenbendazole metabolite, a randomized placebo-controlled first in-human single-dose escalation study was reported in healthy volunteers investigating drug safety and tolerability profiles [19]. Oxifendazole was administered at different doses, and toxicity levels were acceptable without serious adverse events (AEs) or death. However, several subjects experienced hematological or biochemical AEs including leukocytosis, leukopenia, neutropenia, eosinophilia, and changes in bicarbonate and AST levels. In our patient, AST, ALT, and total bilirubin were increased at 1 month after the commencement of oral fenbendazole administration. The antitumor inhibitory effects of fenbendazole on microtubules and RASrelated signaling have been reported; however, the KRAS G12A mutation observed in our NSCLC patient cannot experienced tumor shrinkage on the CT image, and CEA increased slightly. The possibility of liver injury as an immune-related adverse event was also considered, since our patient was also using pembrolizumab. However, after fenbendazole withdrawal, the patient's liver dysfunction spontaneously resolved, and pembrolizumab administration was subsequently resumed. We observed no relapse in hepatic disorder, and therefore we concluded that fenbendazole was the cause of liver dysfunction, although it remained a possibility that pembrolizumab in combination with fenbendazole may have enhanced hepatotoxicity.

Twitter is a major, globally ubiquitous social networking website where messages of up to 140 characters called "tweets" are exchanged between users. In a recent report on cancerrelated tweets, the most common users who tweeted about cancer were a "family member or friend," which accounted for $49.3 \%$ of users [20]. The tweets by a "cancer patient" and "cancer survivor" accounted for $8.7 \%$ each, "news organization/journalist" was $10.1 \%$, and the "healthcare center" was $6 \%$. Another report indicated that the most common conversations related to kidney cancer on Twitter were about support (29.3\%) and treatment (26.5\%) [21]. In our case, the patient and her family received information on fenbendazole from social media sites, after which they purchased the medication and the patient orally self-administered. Interestingly, fenbendazole was marketed and sold as an antiparasitic drug for dogs.

Undoubtedly, patients with advanced cancer and their families desperately seek remedies to fight the disease. Similarly, clinicians are cautious against the use of drugs with limited or

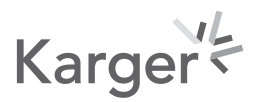


no human safety profiles; the administration of drugs with unproven effects in humans is discouraged because of ethical and safety concerns; however, there are exceptions for investigational drugs in clinical trials. Nonetheless, nonhealthcare professionals cannot make appropriate medical judgments regarding rumors about cancer, and thus may consider unproven drugs as "silver bullets to beat cancer." Based on these observations, physicians should ask patients about their intake of dietary supplements, herbs, or bioactive compounds if unexpected AEs arise.

\section{Conclusion}

Our patient experienced severe hepatic dysfunction after the oral administration of fenbendazole, but no antitumor effects were observed toward her lung cancer. Although fenbendazole has promising antitumor effects, comprehensive clinical trials are required to confirm its pharmacokinetics, safety, and tolerability profiles in humans for future clinical applications.

\section{Acknowledgment}

The authors would like to thank Enago (www.enago.jp) for the English language review.

\section{Statement of Ethics}

Written informed consent was obtained from the patient's son for publication of this case report and any accompanying images because the patient had already died.

\section{Conflict of Interest Statement}

T.H. obtained research grants from Ono Pharmaceutical, Novartis Pharma, Chugai Pharmaceutical, Eli Lilly, Taiho Pharmaceutical, AstraZeneca, Nippon Boehringer Ingelheim, Pfizer, Bristol-Meyers Squibb, Clovis Oncology, Eisai, Takeda Bio, Sumitomo Dainippon Pharma, Abbvie, Merck Serono, MSD, Kyowa Hakko Kirin, Daiichi Sankyo, and Astellas and has received personal fees from Ono Pharmaceutical, Novartis Pharma, Chugai Pharmaceutical, Eli Lilly, Taiho Pharmaceutical, AstraZeneca, Nippon Boehringer Ingelheim, Pfizer, Clovis Oncology, and Bristol-Meyers Squibb. All other authors have no conflicts of interest.

\section{Funding Sources}

No funding was received.

\section{Author Contributions}

Data collection and assembly: T. Yamaguchi and J. Shimizu. Data analysis and interpretation: T. Yamaguchi, J. Shimizu, and Y. Oya. Manuscript writing: T. Yamaguchi. Final manuscript approval: all authors. Accountable for all aspects of the work: all authors. 


\section{Case Reports in Oncology}

\begin{tabular}{l|l}
\hline Case Rep Oncol 2021;14:886-891 \\
\hline DOI: 10.1159/000516276 & $\begin{array}{l}\text { @ 2021 The Author(s). Published by S. Karger AG, Basel } \\
\text { www.karger.com/cro }\end{array}$ \\
\hline
\end{tabular}

Yamaguchi et al.: Liver Injury after the Self-Administration of Fenbendazole

\section{References}

1 Villar D, Cray C, Zaias J, Altman NH. Biologic effects of fenbendazole in rats and mice: a review. J Am Assoc Lab Anim Sci. 2007 Nov;46(6):8-15.

2 Gull K, Dawson PJ, Davis C, Byard EH. Microtubules as target organelles for benzimidazole anthelmintic chemotherapy. Biochem Soc Trans. 1987 Feb;15(1):59-60.

3 Yang X, Ding K. New benzimidazole-2-urea derivates as tubulin inhibitors. Bioorg Med Chem Lett. 2014 Sep 1; 24(17):4250-3.

4 Dogra N, Kumar A, Mukhopadhyay T. Fenbendazole acts as a moderate microtubule destabilizing agent and causes cancer cell death by modulating multiple cellular pathways. Sci Rep. 2018 Aug 9;8(1):11926.

5 Naranjo CA, Busto U, Sellers EM, Sandor P, Ruiz I, Roberts EA, et al. A method for estimating the probability of adverse drug reactions. Clin Pharmacol Ther. 1981 Aug;30(2):239-45.

6 Barr SC, Bowman DD, Heller RL. Efficacy of fenbendazole against giardiasis in dogs. Am J Vet Res. 1994 Jul; 55(7):988-90.

7 Johnston NA, Bieszczak JR, Verhulst S, Disney KE, Montgomery KE, Toth LA. Fenbendazole treatment and litter size in rats. J Am Assoc Lab Anim Sci. 2006 Nov;45(6):35-9.

8 Reichard MV, Wolf RF, Carey DW, Garrett JJ, Briscoe HA. Efficacy of fenbendazole and milbemycin oxime for treating baboons (Papio cynocephalus anubis) infected with Trichuris trichiura. J Am Assoc Lab Anim Sci. 2007 Mar; 46(2):42-5.

9 Mkupasi EM, Sikasunge CS, Ngowi HA, Johansen MV. Efficacy and safety of anthelmintics tested against Taenia solium cysticercosis in pigs. PLoS Negl Trop Dis. 2013 Jul 25;7(7):e2200.

10 Pennisi MG, Hartmann K, Addie DD, Boucraut-Baralon C, Egberink H, Frymus T, et al. Lungworm disease in cats: ABCD guidelines on prevention and management. J Feline Med Surg. 2015 Jul;17(7):626-36.

11 Saini N, Ranjan R, Singla LD, Anand A, Randhawa CS. Successful treatment of pulmonary paragonimiasis in a German shepherd dog with fenbendazole. J Parasit Dis. 2012 Oct;36(2):171-4.

12 Abu-Akkada SS, Oda SS. Prevention and treatment of Encephalitozoon cuniculi infection in immunosuppressed rabbits with fenbendazole. Iran J Vet Res. Spring 2016;17(2):98-105.

13 Lyons ET, Tolliver SC, Ionita M, Collins SS. Evaluation of parasiticidal activity of fenbendazole, ivermectin, oxibendazole, and pyrantel pamoate in horse foals with emphasis on ascarids (Parascaris equorum) in field studies on five farms in Central Kentucky in 2007. Parasitol Res. 2008 Jul;103(2):287-91.

14 Ngomuo AJ, Marriner SE, Bogan JA. The pharmacokinetics of fenbendazole and oxfendazole in cattle. Vet Res Commun. 1984 Aug;8(3):187-93.

15 Marriner SE, Bogan JA. Pharmacokinetics of fenbendazole in sheep. Am J Vet Res. 1981 Jul;42(7):1146-8.

16 Shimomura I, Yokoi A, Kohama I, Kumazaki M, Tada Y, Tatsumi K, et al. Drug library screen reveals benzimidazole derivatives as selective cytotoxic agents for KRAS-mutant lung cancer. Cancer Lett. 2019 Jun 1;451: 11-22.

17 Prichard RK, Kelly JD, Bolin TD, Duncombe VM, Fagan MR. The effect of iron and protein deficiency on plasma levels and parasite uptake of [14C] fenbendazole in rats infected with Nippostrongylus brasiliensis. Aust J Exp Biol Med Sci. 1981 Oct;59(Pt 5):567-73.

18 Short CR, Flory W, Hsieh LC, Barker SA. The oxidative metabolism of fenbendazole: a comparative study. J Vet Pharmacol Ther. 1988 Mar;11(1):50-5.

19 An G, Murry DJ, Gajurel K, Bach T, Deye G, Stebounova LV, et al. Pharmacokinetics, safety, and tolerability of oxfendazole in healthy volunteers: a randomized, placebo-controlled first-in-human single-dose escalation study. Antimicrob Agents Chemother. 2019 Mar 27;63(4):e02255-18.

20 Murthy D, Eldredge M. Who tweets about cancer? An analysis of cancer-related tweets in the USA. Digit Health. 2016 Jul 14;2:2055207616657670.

21 Sedrak MS, Salgia MM, Decat Bergerot C, Ashing-Giwa K, Cotta BN, Adashek JJ, et al. Examining public communication about kidney cancer on twitter. JCO Clin Cancer Inform. 2019 Mar;3:1-6. 